\title{
Increased risk and case fatality rate of pyogenic liver abscess in patients with liver cirrhosis: a nationwide study in Denmark
}

\author{
I Mølle, A M Thulstrup, H Vilstrup, H T Sørensen
}

\begin{abstract}
Background-Patients with liver cirrhosis are at increased risk of serious bacterial infections carrying a high case fatality rate. Case reports have suggested an association between liver cirrhosis and pyogenic liver abscess.

Aims - To estimate the risk and case fatality rate of pyogenic liver abscess in Danish patients with liver cirrhosis compared with the background population.

Methods-Identification of all patients with liver cirrhosis and pyogenic liver abscess over a 17 year period in the National Registry of Patients. Information on death was obtained from the Danish Central Person Registry.

Results-We identified 22764 patients with liver cirrhosis and 665 patients with pyogenic liver abscess, of whom 21 were cirrhotics and 644 were non-cirrhotics. The crude incidence rate of liver abscess in cirrhotics was 23.3 (95\% CI 14.4-35.6) per 100000 person years. The age adjusted risk of liver abscess was increased 15 -fold in patients with cirrhosis compared with the background population. The 30 day case fatality rates in patients with liver abscess and cirrhosis were $38.5 \%$ (13.968.4 ) in alcoholic cirrhosis and $62.5 \%$ (24.5-91.5) in non-alcoholic cirrhosis compared with $26.9 \%(23.5-30.5)$ in liver abscess patients from the background population. After adjustment for sex, age, and comorbidity, the relative risk of death was increased more than fourfold in alcoholic cirrhosis and non-alcoholic cirrhosis compared with the background population.

Conclusions-Liver cirrhosis is a strong risk factor for pyogenic liver abscess associated with a poor prognosis.

(Gut 2001;48:260-263)
\end{abstract}

Keywords: bacterial infections; complications; epidemiology; liver abscess; liver cirrhosis; mortality

Pyogenic liver abscesses are rare in industrialised countries, ${ }^{1-3}$ and the incidence has been estimated at 1.1 per 100000 person years in a Danish regional study. ${ }^{4}$ Biliary disease, malignancy, bacterial infection in other intraabdominal organs, and bacteraemia are factors associated with the occurrence of liver abscess, but the disease is often cryptogenic. ${ }^{24-8}$ However, an association between pyogenic liver abscess and liver cirrhosis has been suggested in case reports, and most often in patients with iron overload. ${ }^{9-12}$ In a few case series of patients with pyogenic liver abscesses, the prevalence of liver cirrhosis was $0.9-13 \%,{ }^{257}$ and the prevalence of chronic alcoholism was more than $10 \%$ in other studies. ${ }^{413}$

To determine if liver cirrhosis is a risk factor for liver abscess, we estimated the incidence rate and 30 day case fatality rate of pyogenic liver abscess in a nationwide cohort of patients with liver cirrhosis referring to the entire Danish population.

\section{Methods}

STUDY POPULATION AND DATA SOURCES

Denmark has approximately 5.2 million inhabitants. Admission, stay, and treatment in Danish public hospitals are free. Records on hospital admissions have been computerised since 1977 in the Danish National Registry of Patients (NRP), and $99.9 \%$ of all discharges from somatic departments in the entire country are recorded here. ${ }^{14}$ Each admission record includes the CPR number (see below), date of admission, date of discharge, and up to 20 discharge diagnoses.

Every Danish citizen has been recorded in the Central Person Registry (CPR) since 1968, using a personal identification number (CPR number) for each citizen. The CPR number is assigned at birth or immigration, and includes date of birth and an additional number carrying a code for sex.

The study was based on record linkage between two nationwide population based cohorts from the NRP. The first cohort included patients with liver cirrhosis and the second patients with pyogenic liver abscess. Diagnosis codes according to the Danish version of the International Classification for Diseases, 8th edition (ICD-8), were used for primary identification in the NRP. ${ }^{15}$ Dates of death or emigration were obtained in the CPR.

IDENTIFICATION OF PATIENTS WITH LIVER CIRRHOSIS

The inclusion criterion for this study cohort was at least one discharge with a diagnosis of liver cirrhosis: alcoholic cirrhosis (ICD$8=571.09$ ), primary biliary cirrhosis (571.90),

Abbreviations used in this paper: $\mathrm{CPR}$, Danish Central Person Registry; CPR number, personal identification number; NRP, Danish National Registry of Patients; ICD-8, International Classification of Diseases, 8th edition; IR, incidence rate; IRR, incidence rate ratio; SIR, age standardised incidence ratio. 
Table 1 Characteristics of patients with liver cirrhosis in the National Registry of Patients in Denmark, 1977-93

\begin{tabular}{|c|c|c|c|c|c|}
\hline & \multicolumn{2}{|c|}{ Alcoholic cirrhosis } & \multicolumn{2}{|c|}{ Non-alcoholic cirrhosis } & \multirow[b]{2}{*}{ All } \\
\hline & Men & Women & Men & Women & \\
\hline No of patients & 10001 & 3874 & 4224 & 4665 & 22764 \\
\hline Cases with liver abscess & 9 & 4 & 3 & 5 & 21 \\
\hline Mean age at entry (y) & 53.7 & 53.7 & 61.4 & 64.4 & 57.4 \\
\hline Mean follow up (y) & 4.1 & 4.6 & 3.0 & 4.1 & 4.0 \\
\hline Person years at risk & 40635 & 17683 & 12667 & 19175 & 90160 \\
\hline $\begin{array}{l}\text { IR per } 100000 \text { years } \\
\quad(95 \% \mathrm{CI})\end{array}$ & $\begin{array}{c}22.2 \\
(10.1-42.0)\end{array}$ & $\begin{array}{c}22.6 \\
(6.2-57.9)\end{array}$ & $\begin{array}{c}23.7 \\
(4.9-69.2)\end{array}$ & $\begin{array}{c}26.1 \\
(8.5-60.9)\end{array}$ & $\begin{array}{c}23.3 \\
(14.4-35.6)\end{array}$ \\
\hline
\end{tabular}

IR, incidence rate.

non-specified cirrhosis (571.92), chronic hepatitis (571.93), or other types of cirrhosis, alcoholism not indicated (571.99) from 1 January 1977 to 31 December 1993. The Danish version of ICD-8 makes it possible to modify the diagnoses using a sixth digit as a suffix to the diagnosis code, indicating for instance observation for, sequel, or earlier. As we had no access to the primary hospital records, yet we wanted to achieve the lowest possible rate of misclassification, we excluded all individuals with these modifications. Individuals less than 20 years of age were also excluded as liver cirrhosis is rare in this age group in Denmark and is usually associated with congenital malformations or inborn errors of metabolism.

The cohort was divided into two subcohorts: alcoholic liver cirrhosis and non-alcoholic liver cirrhosis. Cirrhotics with a diagnosis of alcoholism (ICD-8=303) at any time during the follow up period were always referred to the alcoholic subcohort, independent of the code for cirrhosis. The purpose of this was to reduce misclassification between the two subcohorts. $^{16}{ }^{17}$

IDENTIFICATION OF CASES OF LIVER ABSCESS During the follow up period from 1 January 1977 to 31 December 1993, all cases of liver abscess were identified in the NRP, which was searched for the diagnosis codes for pyogenic liver abscess (572.01) and suppurative hepatitis (572.09). Cases with amoebic liver abscess were not included. Individuals with any modification of the diagnosis codes were also excluded from this cohort.

STATISTICAL ANALYSES

In the liver cirrhosis cohort, the follow up period started at the date of discharge from hospital with a diagnosis of cirrhosis and ended at the date of discharge with a diagnosis of liver abscess, date of death, date of emigration, or 31 December 1993, whichever came first.

The association between liver cirrhosis and liver abscess was notified only when the diagnosis of liver cirrhosis was made before or during the same admission as the diagnosis of pyogenic liver abscess. The purpose of this was to associate the diseases only if patients actually had liver cirrhosis when admitted with liver abscess. The follow up period for the background population was based on the number of inhabitants $\geqslant 20$ years of age in Denmark for each year of the follow up period.

Incidence rate (IR) was calculated as the number of cases divided by the follow up time for each type of cirrhosis and sex. The incidence rate ratio (IRR) of liver abscess in the cirrhosis cohort was estimated by dividing the IR in the alcoholic cirrhosis subcohort by the IR in the non-alcoholic subcohort. The standardised incidence rate (SIR) of liver abscess was based on the age specific IR values in the cirrhosis cohort and in the background population, using 20 year age groups in sex specific strata.

Confidence intervals of $95 \%$ were estimated according to exact limits when expected numbers were less than 10 or otherwise according to the Poisson distribution. The expected numbers of cases of liver abscess in the cirrhosis cohort were estimated by multiplying the number of person years in cirrhotics by the IR found in the background population.

The relative risk (odds ratio) of 30 day case fatality in patients with liver abscess and liver cirrhosis adjusted for sex, age, and comorbidity was estimated using logistic regression models. ${ }^{18}$ The cohort was subdivided according to age: less than 55 years (the reference category) and older than 55 years. The comorbidity index was estimated as described by Charlson and colleagues. $^{19}$ Liver diseases were not scored, and only discharge diagnoses registered at the same admission for pyogenic liver abscess were scored.

\section{Results}

The cirrhosis cohort comprised 22764 individuals (table 1). In both subcohorts there was a shorter mean follow up period for men, and there was a considerably larger proportion of alcoholic men than women in the cohort. Sixty one percent of all patients with cirrhosis were alcoholics, and in these patients cirrhosis was diagnosed on average 8-10 years earlier than non-alcoholics, according to their age (table 1).

The liver abscess cohort comprised 665 individuals, of whom 21 also had a diagnosis of liver cirrhosis.

The overall IR of pyogenic liver abscess in the liver cirrhosis cohort was 23.3 (14.6-35.6) per 100000 person years (table 1 ). The overall IR of liver abscess in the background population (cirrhosis cohort not included) was 1.0 (0.9-1.1).

The increased risk of liver abscess in cirrhotics compared with the background population is shown in table 2. The overall age SIR was 15.4 (9.6-23.6). The SIRs of liver abscess in patients with alcoholic and non-alcoholic liver cirrhosis were $15.5(8.2-26.5)$ and 15.7 (6.8-30.9) respectively, compared with the background population. However, the magnitude of the estimates changed according to age as the incidence rate ratios of liver abscess (IRR) in alcoholic cirrhotics and non-alcoholic cirrhotics differed significantly in the two age groups: $3.3(0.5-143.1)$ versus $0.4(0.1-1.6)$ in cirrhotics younger and older than 55 years, respectively. Thus the SIRs in the two cirrhosis groups (table 2) should be related to the background population and not to each other. The first year after the diagnosis of liver cirrhosis was associated with the highest SIR for pyogenic liver abscess (36.4) but during the 
Table 2 Observed and expected numbers and age standardised incidence rates of liver abscess in 22764 patients with liver cirrhosis

\begin{tabular}{lcllr}
\hline & Observed & Expected & SIR $^{1}$ & \multicolumn{1}{c}{$95 \%$ CI } \\
\hline All cirrhotics $^{2}$ & 21 & 1.4 & 15.4 & $9.6-23.6$ \\
Alcoholic cirrhosis $^{2}$ & 13 & 0.8 & 15.5 & $8.2-26.5$ \\
Non-alcoholic cirrhosis $^{2}$ & 8 & 0.5 & 15.7 & $6.8-30.9$ \\
Women $^{3}$ & 9 & 0.5 & 18.0 & $8.2-34.2$ \\
Men $^{3}$ & 12 & 0.9 & 14.0 & $7.2-24.4$ \\
First year after cirrhosis diagnosis $^{2}$ & 8 & 0.2 & 36.4 & $15.7-71.6$ \\
Subsequent follow up time $^{2}$ & 13 & 1.1 & 11.8 & $6.3-20.2$ \\
\hline
\end{tabular}

${ }^{1}$ Age standardised incidence rate.

${ }^{2}$ Reference to the background population, cirrhosis cohort excluded.

${ }^{3}$ Reference to the sex specific strata in the background population, cirrhosis patients excluded.

Table 3 The risk of 30 day case fatality in patients with liver abscess in Denmark

\begin{tabular}{|c|c|c|c|c|}
\hline & $\begin{array}{l}\text { Deaths/ } \\
\text { No of cases }\end{array}$ & $\begin{array}{l}\text { Crude } \\
\text { odds ratio }\end{array}$ & $\begin{array}{l}\text { Adjusted } \\
\text { odds ratio }\end{array}$ & $95 \% C I$ \\
\hline \multicolumn{5}{|l|}{ General } \\
\hline Background population ${ }^{1}$ & $173 / 644$ & 1.0 & 1.0 & - \\
\hline Alcoholic cirrhosis & $5 / 13$ & 1.7 & 4.3 & $1.2-15.2$ \\
\hline Non-alcoholic cirrhosis & $5 / 8$ & 4.5 & 4.8 & $1.0-22.4$ \\
\hline \multicolumn{5}{|l|}{ Comorbidity index } \\
\hline 0 score $^{1}$ & $97 / 475$ & 1.0 & 1.0 & - \\
\hline 1 score & $27 / 87$ & 1.8 & 1.4 & $0.8-2.4$ \\
\hline 2 score & $38 / 71$ & 4.5 & 3.9 & $2.3-6.8$ \\
\hline$>2$ score & $21 / 32$ & 7.4 & 8.7 & $3.9-19.7$ \\
\hline \multicolumn{5}{|l|}{ Age } \\
\hline$\geqslant 20<55 \mathrm{y}^{1}$ & $21 / 191$ & 1.0 & 1.0 & - \\
\hline$\geqslant 55 \mathrm{y}$ & $162 / 474$ & 4.2 & 3.9 & $2.3-6.7$ \\
\hline \multicolumn{5}{|l|}{ Sex } \\
\hline $\operatorname{Men}^{1}$ & $76 / 367$ & 1.0 & 1.0 & - \\
\hline Women & $107 / 298$ & 2.1 & 2.0 & $1.3-2.9$ \\
\hline
\end{tabular}

${ }^{1}$ Data from the entire Danish population without cirrhosis cohort.

subsequent follow up period there was also a considerably increased risk of liver abscess in the cirrhosis cohort compared with the background population (table 2).

The overall 30 day case fatality rate in patients with liver abscess was 38.5\% (13.968.4) for patients with alcoholic cirrhosis and $62.5 \%(24.5-91.5)$ for patients with nonalcoholic cirrhosis. A somewhat lower case fatality rate of $26.9 \%(23.5-30.5)$ was found among other patients with pyogenic liver abscess. After adjustment for age, sex, and comorbidity, both groups of cirrhotics had a more than fourfold higher risk of dying within 30 days than other patients with pyogenic liver abscess (table 3). There was however no significant difference between the two cirrhosis groups. Female sex and age greater than 55 years were also associated with an increased risk of dying in the liver abscess cohort. A high comorbidity index was a strong prognostic factor for death after diagnosis of pyogenic liver abscess (table 3).

\section{Discussion}

In this nationwide population based study, we found a considerably increased risk of pyogenic liver abscess in patients with liver cirrhosis compared with the background population. We also found that liver abscess associated with cirrhosis increased the risk of dying within 30 days after admission more than fourfold compared with other patients with liver abscess.

Our registry based study design has strengths and limitations. The uniformly structured Danish health care system and the NRP enabled us to conduct a large population based study with a complete follow up. Our registry based estimate of the incidence rate of liver abscess in the background population is in close agreement with earlier findings in a Danish regional study in which several data collection methods were used. ${ }^{4}$

Misclassification of discharge diagnosis codes, a well known methodological problem in registry based research, is probably less than $12 \%{ }^{4}{ }^{16}$ Any non-differential misclassification would tend to underestimate the association found in this study. Our data lack clinical details on patients. Increased hospitalisation and extensive diagnostic efforts in cirrhotics could partly explain the association and is probably responsible in part for the high risk of pyogenic liver abscess found during the first year after diagnosis of liver cirrhosis (table 2). However, given the magnitude of the association and the lack of compensatory effect in the prognosis, the association between liver cirrhosis and pyogenic liver abscess found in the present study cannot be explained solely in terms of this bias.

Patients with liver cirrhosis have reduced transhepatic blood flow, increased portal pressure, and often ascites, which may compromise immune defences and increase intestinal permeability to bacteria. The macrophage function is impaired, ${ }^{20}{ }^{21}$ and Kupffer cells are therefore probably less protective; the functions of neutrophilic leucocytes ${ }^{22}$ and the complement system ${ }^{24}$ are often impaired in liver cirrhosis. Furthermore, in the cirrhosis cohort there was a mean follow up time of only four years, undoubtedly due to a high mortality rate, which indicates that many cirrhotics were diagnosed late in the disease course where immunodysfunction is prominent. These relations may explain the increased risk of liver abscess in cirrhotics.

After stratification for age, the risk of pyogenic liver abscess in the alcoholic and non-alcoholic cirrhosis subcohorts varied over strata, which did not allow us to compare the risks for liver abscess in the two subcohorts. But we did find a substantially increased age adjusted risk of pyogenic liver abscess in both subcohorts compared with the background population. Therefore, the aetiology of liver cirrhosis is probably not a crucial factor for the increased risk of pyogenic liver abscess in cirrhosis. Cirrhosis itself seems to be an important risk factor for pyogenic liver abscess but nevertheless immunosuppresion caused by alcohol $^{25}$ is probably another important risk factor. $^{413}$

The short term prognosis in the liver abscess cohort was considerably worse in cirrhotics than in other patients, and we conclude that liver cirrhosis is a strong risk factor for liver abscess associated with a poor prognosis. This is probably due to impaired immune defences and structural changes in the cirrhotic liver.

The study was supported by the Danish Medical Research Council (grant No 97700677).

1 Frey CF, Zhu Y, Suzuki M, et al. Liver abscesses. Surg Clin North Am 1989;69:259-71. 
2 Pitt HA, Zuidema GD. Factors influencing mortality in the treatment of pyogenic hepatic abscess. Surg Gynecol Obstet $1975 ; 140: 228-34$

3 Miedema BW, Dineen P. The diagnosis and treatment of pyogenic liver abscesses. Ann Surg 1984;200:328-35.

4 Hansen PS, Schønheyder HC. Pyogenic hepatic abscess. A 10 -year population-based retrospective study. APMIS 1998;106:396-402.

5 Huang CJ, Pitt HA, Lipsett PA, et al. Pyogenic hepatic abscess. Changing trends over 42 years. Ann Surg 1996;223:600-9.

6 Chu KM, Fan ST, Lai EC, et al. Pyogenic liver abscess. An audit of experience over the past decade. Arch Surg 1996;131:148-52.

7 Chou FF, Sheen-Chen SM, Chen YS, et al. Prognostic factors for pyogenic abscess of the liver. 7 Am Coll Surg 1994; 179:727-32.

8 Hashimoto L, Hermann R, Grundfest-Broniatowski S. Pyogenic hepatic abscess: results of current management. Am Surg 1995;61:407-11.

9 Leyman P, Baert AL, Marchal G, et al. Ultrasound and CT of multifocal liver abscesses caused by Yersinia enterocolitica. F Comput Assist Tomogr 1989;13:913-15.

10 Scully R, Galdabini J, McNeely B. Case records of the Massachusetts General Hospital. N Engl f Med 1978;299:81926.

11 Scully R, McNeely B. Case records of the Massachusetts General Hospital. N Engl f Med 1975;292:963-8.

12 Strungs I, Farrell DJ, Matar LD, et al. Multiple hepatic abscesses due to Yersinia enterocolitica. Pathology 1995;27: $374-7$

13 Seeto RK, Rockey DC. Pyogenic liver abscess. Changes in etiology, management, and outcome. Medicine (Baltimore) 1996;75:99-113.
14 The activity in the hospital care system, 1984. Copenhagen: Danish National Board of Health, 1985:49-59.

15 Classification of diseases. Copenhagen: Danish National Board of Health, 1986

16 Vestberg K, Thulstrup AM, Sørensen HT, et al. Data quality of administratively collected hospital discharge data for liver cirrhosis epidemiology. F Med Syst 1997;21:11-20.

17 Almdal TP, Sørensen TI. Incidence of parenchymal liver diseases in Denmark, 1981 to 1985: analysis of hospitalization registry data. The Danish Association for the Study of the Liver. Hepatology 1991;13:650-5.

18 Hosmer DW, Lemeshow S. Applied logistic regression. New York: John Wiley and Sons, 1989.

19 Charlson ME, Pompei P, Ales KL, et al. A new method of classifying prognostic comorbidity in longitudinal studies: development and validation. F Chronic Dis 1987;40:37383.

20 Holdstock G, Leslie B, Hill S, et al. Monocyte function in cirrhosis. f Clin Pathol 1982;35:972-9.

21 Rimola A, Soto R, Bory F, et al. Reticuloendothelial system phagocytic activity in cirrhosis and its relation to bacterial infections and prognosis. Hepatology 1984;4:53-8.

22 Laffi $G$, Carloni V, Baldi $\mathrm{E}$, et al. Impaired superoxide anion, platelet-activating factor, and leukotriene B4 synthesis by neutrophils in cirrhosis. Gastroenterology 1993;105:170-7.

23 Rajkovic IA, Williams R. Mechanisms of abnormalities in Rajkovic IA, Williams R. Mechanisms of abnormalities in
host defences against bacterial infection in liver disease. host defences against bacte
Clin Sci 1985;68:247-53.

24 Homann C, Varming K, Hogasen K, et al. Acquired C3 deficiency in patients with alcoholic cirrhosis predisposes to infection and increased mortality. Gut 1997;40:544-9.

25 MacGregor RR. Alcohol and immune defense. $7 A M A$ 1986;256:1474-9. 